Research Article

\title{
Teaching System of Undergraduate Entrepreneurship Education under the Background of Internet of Things
}

\author{
Heng Zhang' and Xiubo Liu (iD) ${ }^{2}$ \\ ${ }^{1}$ Xi'an Fanyi University, Xi'an 710100, Shaanxi, China \\ ${ }^{2}$ Dean's Office, Jilin Engineering Normal University, Changchun 130052, Jilin, China \\ Correspondence should be addressed to Xiubo Liu; 1xb7227@jlenu.edu.cn
}

Received 27 August 2021; Revised 12 October 2021; Accepted 26 October 2021; Published 17 November 2021

Academic Editor: Sang-Bing Tsai

Copyright ( $\odot 2021$ Heng Zhang and Xiubo Liu. This is an open access article distributed under the Creative Commons Attribution License, which permits unrestricted use, distribution, and reproduction in any medium, provided the original work is properly cited.

\begin{abstract}
This article mainly introduces the research on the teaching system of entrepreneurship education for college students under the background of the Internet of Things. On the basis of extensive investigation and analysis, this article combines the reality of entrepreneurship education in our country's universities to study how to construct an entrepreneurial education system. This paper designs the social support guarantee and specific implementation plan for corporate education by constructing the corporate goal system, curriculum system, practical curriculum system, training team, and other systems, defines the relevant concepts of the innovation and entrepreneurship education system, and analyzes the related concepts of innovation and entrepreneurship education. Theories provide a theoretical basis for constructing college students' innovation and entrepreneurship education system. Based on the analysis and construction of the innovation and entrepreneurship education system for college students in our country, the necessity and feasibility of the innovation and entrepreneurship education system for college students are further elaborated. The experimental results of this paper show that after the theoretical viewpoints and operational content of the EE system are put forward, the entrepreneurial enthusiasm of college students has increased by $15 \%$, the learning ability has been significantly improved, and it has also fully demonstrated the teaching effect of the entrepreneurial education teaching system for college students under the background of the Internet of Things. In addition, their understanding of EE and teaching has also improved significantly. This structure provides an important reference for further research and practice of undergraduate EE.
\end{abstract}

\section{Introduction}

The development of innovation and entrepreneurship education is not the negation of old things by new things, but the inheritance and development. Innovation and entrepreneurship education is the product of the times, developed on the basis of creativity education, innovation education, and entrepreneurship education. At present, generally speaking, the understanding of entrepreneurial connotation can be divided into broad sense and narrow sense [1]. The common understanding of entrepreneurship at home and abroad is that entrepreneurship is to start a business, which is a narrow understanding of entrepreneurship [2]. Entrepreneurship in a narrow sense can be innovative behavior or imitative behavior, as long as it is based on the needs of the society to carry out production and business activities, and entrepreneurs create their own enterprises, which can be called entrepreneurship. Entrepreneurship in a broad sense includes not only the behavior of starting a company but also the behavior of staff to develop and innovate in their own work, covering a wider range, including every link of the work process.

The connection and communication between things and the realization of information sharing are the characteristics of the IoT [3]. This is different from the interconnection communication mode of machines and machines realized by the Internet, the interconnection communication mode between people and people realized by the mobile communication network, and the IoT. The network transmits logistics information, and its network entity is the physical world, so its application range will be broader and will have a 
more profound impact on human society [4]. As a powerful technology today, the Internet of Things has been applied to all walks of life, among which teaching through the background of the Internet of Things can get better teaching results. Based on the characteristics of the Internet of Things, this article takes the Internet of Things system architecture as the core and builds the Internet of Things teaching system structure. The curriculum system structure can ensure that the training of students' abilities is more reasonable, systematic, and orderly and can ensure the teaching content and teaching methods.

The Internet of Things (IoT) is a dynamic global information network composed of objects connected to the Internet, which have become an integral part of the Internet in the future [5]. Perera $\mathrm{C}$ surveyed more than one hundred IoT smart solutions on the market and carefully checked them to identify the technologies, functions, and applications used. However, due to the fact that there are many unstable factors in the survey, the results will not be so accurate [6]. Hasegawa $\mathrm{T}$ has developed a full-color LED display with a color sensor function [7]. The display has a new function that can draw pictures and text by shining light. However, due to the gap between the color changes, the result is not very accurate [8]. The comprehensive study by Liao $\mathrm{H}$ examines the reporting use of credible techniques in higher education evaluation articles that use qualitative methods. However, due to some unstable factors in the article, the results are not very accurate [9].

The innovation of this article lies in the following: (1) Based on the idea of "promoting the all-round development of college students," it constructs the innovation and EE system for college students from the aspects of target system, knowledge system, support and guarantee system, practice platform, and evaluation system. It does not only focus on college students. The single element of the innovation and EE system is different from many previous studies. (2) Based on the architecture of the IoT, through the analysis of the various components and application scenarios of the IoT, an IoT network protocol based on the general functions of the IoT is designed, and the main business processes in the IoT application are proposed. A detailed analysis and elaboration are carried out, which will provide good guidance in specific practical applications.

\section{Research Methods of EE and Teaching System under the Background of IoT}

\subsection{Research Methods of Undergraduate EE}

2.1.1. Historical Analysis Method. Innovation and EE are the latest educational concept and education model. Through historical analysis, it explains the evolution of our country's college education from "innovation education-EE-innovation and EE" [10].

2.1.2. Comparative Analysis Method. After comparing the innovation and EE of Chinese college students with that of foreign college students, we can find the shortcomings of our universities and learn the advanced practices of foreign universities [11].

2.1.3. Statistical Research Method. Statistical analysis was performed on the data collected in the previous questionnaire. This study mainly uses descriptive statistics and cross analysis. Through frequency statistics and ratio analysis, we can understand the proportions of various elements in the innovation and EE of college students and use cross analysis to analyze the characteristics of different demographics. The situation of student innovation and EE is compared $[12,13]$.

2.2. IoT Technology. There are a large number of nodes in the Internet of Things, and these nodes need positioning technology to understand the distribution of nodes and better optimize the network [14].

The development of positioning technology is constantly improving with changes in hardware conditions, network models, market requirements, and so on [15]. For example, in an environment with good hardware support, with strong computing power and better storage capabilities, higher complexity can be achieved if the hardware conditions do not allow it; you must design an algorithm with low time and space complexity. The network model is also an important factor in determining the positioning algorithm. The current network structure includes star, tree, and Mesh Network, and other structures [16]. Different network structures also determine the selection and specific implementation of positioning algorithms; market demand is the eternal driving force to promote the development of positioning technology [17]. In different application scenarios, positioning functions are required, and different environments are required. Medium positioning requirements are different. For example, in indoor positioning, the positioning algorithm error must be controlled within a reasonable range. Compared with outdoor positioning, the absolute positioning error must meet the indoor positioning requirements [18].

The IoT is a new type of network that gathers a large number of nodes [19], and the positioning technology in the IoT needs to consider the actual characteristics of the network and the selection of equipment for positioning algorithms, especially the large number of nodes, cost, processing capacity, and power consumption. It is the key factor that restricts the network, so the positioning function of the network cannot be realized through GPS positioning [20].

Positioning technology is of great significance in IoT applications. First, the location information can provide the location distribution relationship of each node in the network. If the location information of the nodes in the network can be understood, then the selection of network routing will become easier, and the best route selection between two nodes can be realized [21] so as to realize the simplification of network protocol and save the transmission energy of network nodes [22]. Power consumption is a key indicator in the IoT, and it is also a core factor affecting the practical application of the IoT. When it is popularized on a large 
scale, low power consumption must be achieved. In the IoT system, positioning technology can better optimize the network. The structure and transmission route have a good effect on reducing power consumption [23]. Second, the location information realized by positioning technology is also a practical requirement in many applications. For example, the application needs to know the specific location of the node, the location information of the event, and so on, so this information can be used for further operations [24, 25].

\subsection{EE Methods in the Context of the IoT}

2.3.1. Establishing an Evaluation System for EE. The introduction of an entrepreneurial education evaluation system in the context of the IoT is an assessment method that has developed to a certain extent. This is also the last link of EE, which can make a more comprehensive evaluation of EE [26]. Therefore, when inspecting students, we must not only understand their reading ability but also examine their application ability, the number of companies established, the number of jobs, and the success rate of the project. All indicators can be reported [1]. At the same time, they must be observed and evaluated. By observing their performance in commercial projects, assess their business capabilities and overall quality [27]. The second is to establish a differentiated evaluation system [28]. Each evaluation question belongs to a different part, and EE can be evaluated from multiple aspects. However, government power is relatively high and can be used as the core to measure local economic activities [29]. The main purpose of innovation and entrepreneurship education is to cultivate students' entrepreneurial and innovative abilities. Therefore, when examining students, we must not only understand their mastery of book knowledge but also look at their ability to use, the number of companies founded, the number of positions, and the success rate of projects. All indicators can be referred to, and at the same time, they must observe and assess their performance in entrepreneurial projects to judge their entrepreneurial ability and overall quality.

\subsubsection{Establishing a Diversified Entrepreneurial Education} Model. The EE system has the characteristics of multifaceted assessment capabilities, which can help college students in the development of various aspects of entrepreneurial learning, which is of considerable help. The innovation and EE system is to innovate the EE model, no longer follow the education model of the first and second departments, and classify the training of talents: low-level students must pay attention to professional knowledge and humanistic knowledge education [30]. Middle-level and upper-level students can divide courses according to their personal wishes, allowing them to learn business knowledge in this field according to their personal preferences. Students who are about to graduate will be trained according to their employment intentions [31, 32]. If they choose entrepreneurship as a career, entrepreneurship training should be strengthened to help them realize the connection between the incubation base and market practice [33]. Of course, not everyone is suitable for entrepreneurship. When students have entrepreneurial ideas, the instructors of the entrepreneurship training team can put forward reasonable suggestions based on the students' actual skills and family conditions and inform them of the risks they may face during the business period; in this way, students can have a general idea and can calmly deal with the problems they face. Colleges and universities support and encourage students to start their own businesses, especially to improve their comprehensive ability, so that they can prepare for entering society as soon as possible and accept changes in life and identity with confidence [34].

\section{Teaching Experiment of EE under the Background of IoT}

\subsection{IoT System Framework}

3.1.1. Composition of the IoT Transmission Network. The macroperspective mainly focuses on the overall components of the IoT system and uses a macrodescription to summarize the composition of the IoT system. The IoT is mainly composed of three parts: the network terminal part, the transmission network part, and the data processing center (application layer) part.

The network terminal part of the first part refers to the sensor, also known as the collection front end. This is the final source of the data of the IoT. This is also the perception layer of the IoT. It uses sensors to sense or collect different external signals to achieve data collection and processing, the conversion becomes the signal that people need, and in most cases, it is an electrical signal [35]. Afterward, corresponding data processing is performed according to the corresponding relationship between the external signal and the electrical signal. There are high requirements for the sensor. In different environments, the requirements of the sensor are different according to the actual application. For example, when measuring the $\mathrm{PH}$ value of water, the sensor needs to be isolated and protected, and the blood pressure and electrocardiogram of the human body are measured. In the case of physiological parameters, it is necessary to design protective measures to prevent harm to the body [36]. At the same time, low power consumption, small size, stability, long life, and low price are all specific requirements of the network terminal.

The second part is the transmission network part, which is mainly responsible for the role of transmitting information; that is, the various data collected by the network terminal part are transmitted through this transmission network, from the detection point to the corresponding management control center. Due to the wide variety of network terminals and many deployment areas, it is necessary to make this transmission network stable and efficient in various applications.

Data transmission requires people to design a transmission network with high stability coefficient, strong interference processing capability, and large network capacity [37].

The third part is the control processing center part. The transmission network part transports the data collected 
from the front-end part. The control processing center will classify and integrate these data. According to the purpose of the application, the effective data will be screened out, and abnormal data will be eliminated. The processing algorithm obtains the processing result. In some applications, the result should be transmitted through the abovementioned reverse communication path and sent to the network terminal part, thus realizing a two-way communication between the control center and the overall network. The data processing center is based on the first two parts, so it must be designed with full reference to the scale and characteristics of the first two parts to be able to carry out reasonable and efficient data control and processing in practical applications.

Components of the IoT Terminal. The general component modules of the IoT network terminal are derived from the basic requirements and general functional requirements of the terminal. According to the general functions analyzed above, the various component modules of the terminal will be described in detail as follows.

The network terminal of the IoT mainly includes the following functional modules:

(1) MCU module, which is the core processing part of the whole module, mainly realizes data processing and centralized control of the system. However, due to cost and other factors, MCUs with faster processing speeds cannot be used when selecting models, and low power consumption requirements must be met. This has been paid enough attention in the current MCU industry. For example, the MSP430 series of Texas Instruments is a good choice; the official has also introduced more application schemes, which can provide a good technical reference in actual design applications [38].

(2) Data storage module: this module is mainly responsible for the storage and management of data. The main function realized is the screening and storage of key data, such as simple user information and key collected data. The purpose of the data storage is mainly for general query and assisting in error correction and retransmission.

(3) External interface module: this module is a means for network terminals to communicate with external devices (PC and other terminals). Common external interface modules include serial ports, USB, and SPI.

(4) Sensor module: this module is the core realization part of the entire terminal function to collect the corresponding data, filter, code, convert, and finally, become usable effective data. Common sensor modules are temperature sensor, humidity sensor, pressure sensor, $\mathrm{PH}$ sensor, and so on. The working cycle of the sensor module is also different. There are intermittent collections and continuous collections. For example, the blood pressure sensor must work continuously, while the temperature sensor needs a certain time interval for data measurement.
The main professional courses of the Internet of Things in the application layer are professional courses based on programming technology and database principles, combined with computer operating systems, information security technology, software engineering, curriculum design, comprehensive practice, and so on, with related technologies at the perception layer and transmission layer core professional courses.

\subsection{Construction of the EE Teaching System. The innovation} and EE system for college students refers to an organic whole that integrates the various elements of the innovation and EE for college students and is a system converged by a variety of social resources and policy environments. This paper takes the goal system of college students' innovation and EE as the core, focuses on the knowledge system and practice platform of innovation and EE, relies on the service guarantee platform, evaluation mechanism, and other aspects, and scientifically builds a multiparty linkage of "university, government, enterprise, society, and students" innovation and EE system for college students. The details are shown in Figure 1.

Innovation and entrepreneurship education is mainly to strengthen college students' intake of entrepreneurial knowledge and improve their comprehensive quality and innovation and entrepreneurship capabilities. These stages influence each other. Colleges and universities need to actively explore new teaching mechanisms, choose different teaching systems according to different educational objects, establish brand-new teaching models, and find new ideas for the creation of teaching systems. As the foundation of all elements of the education system, educational goals have an extraordinary position. When the goal of innovation and EE for college students is clear, the content of education can be selected around the goal, suitable education methods, and models, and necessary support and guarantee can be provided. Generally speaking, the goal of innovation and EE for college students is not only to allow more college students to choose entrepreneurship but also to establish a concept of innovation and EE, to organically combine innovation and EE with ideological and political education, and to give full play to the education and education of ideological and political education.

\section{Analysis of the Teaching System of Undergraduate EE in the Context of the IoT}

4.1. Analysis of the Impact of the IoT on College Students' EE. The influence of the IoT on the elements of EE for college students: The IoT has spawned a large number of IoT companies and online education platforms, which have enriched the constituent elements of the entrepreneurial ecosystem and injected fresh vitality into the entrepreneurial ecosystem. The IoT promotes the dissemination of culture and knowledge, expands the entrepreneurial population, and improves the openness of industry information and work efficiency. The specific results are shown in Table 1. 


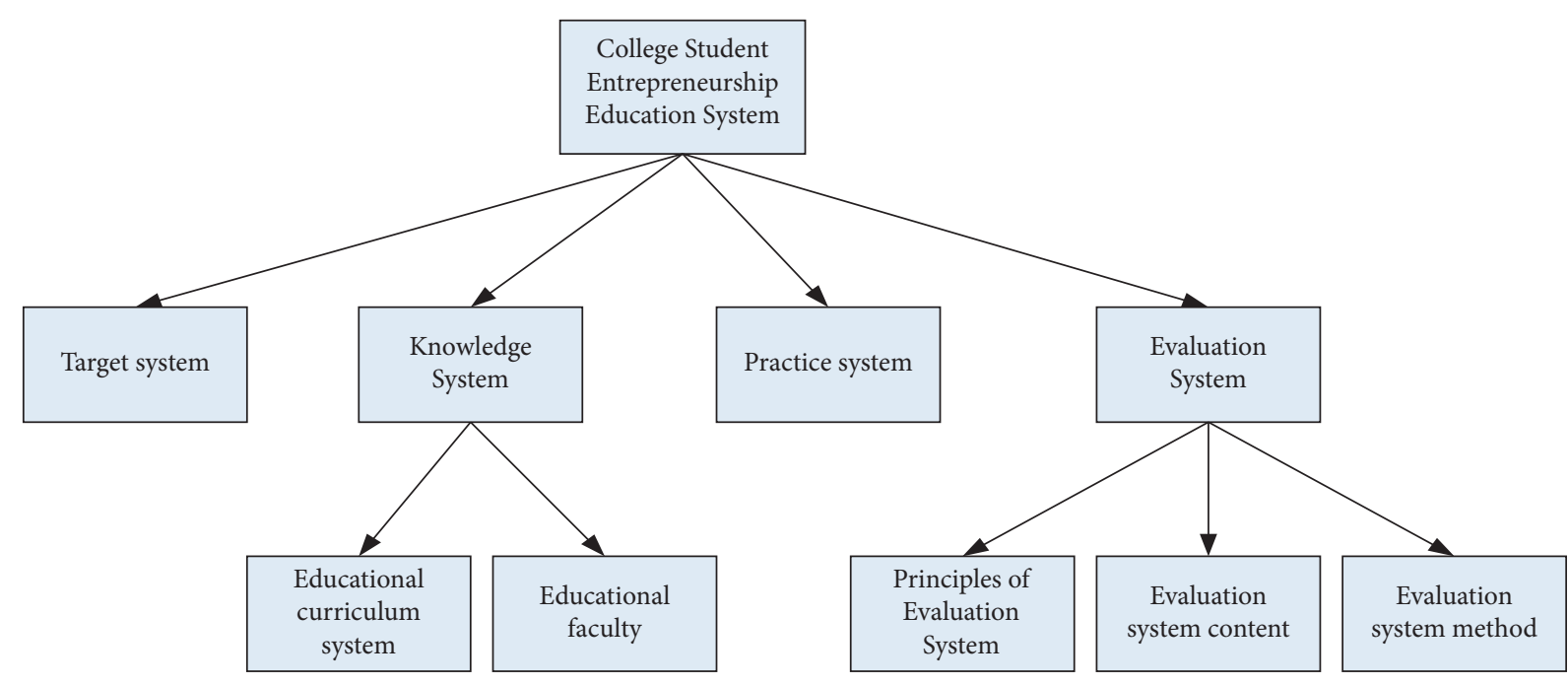

FIgURE 1: Teaching system structure of EE.

TABLE 1: Results of the impact of the IoT on college students' EE.

\begin{tabular}{|c|c|c|c|c|c|}
\hline Influences & Cultural communication & Entrepreneurial crowd & Industry information & Work efficiency & Positivity \\
\hline Before & $23 \%$ & $24 \%$ & $14 \%$ & $26 \%$ & $9 \%$ \\
\hline After & $43 \%$ & $32 \%$ & $34 \%$ & $33 \%$ & $27 \%$ \\
\hline
\end{tabular}

In order to more intuitively observe the impact of the IoT on the entrepreneurial education of college students, the table is drawn into a graph, as shown in Figure 2.

In terms of the integration and flow mechanism of system resources, the IoT has a positive impact on reducing operating costs and entrepreneurial difficulties, helping to promote integration and the flow of talent, capital, materials, technology, and information. In terms of the system's value exchange mechanism, operators should use the Internet to integrate resources, provide value, lower the threshold for entrepreneurship, and further intensify competition among enterprises. In the commercial market, there is also a development model; that is, enterprises realize "survival and profit" through resource allocation. In terms of the mechanism to adapt to system feedback, the state has promoted the integration of mobile Internet, big data, and so on, with modernization. In recent years, in accordance with market laws, the response of the entrepreneurial market has accelerated, and the pressure of competition has increased. The flow of resources and the efficiency of resource utilization have also been promoted and improved to a certain extent for new enterprises that are most suitable for survival. Regarding the cooperative development mechanism of the system, many functions of the business education ecosystem can be realized through the combination of online and offline. In the context of the IoT, the mechanism has been continuously strengthened, and the scale expansion and operation improvement speed have been significantly accelerated.

China's economic development can also benefit from it. The IoT is an emerging thing. Colleges and universities can use practice bases to train students for thinking divergence, improve students' practical ability, and come up with new ideas. Third, the school can hold entrepreneurial practice competitions so that students can learn teamwork in actual combat and understand the importance of creativity and cooperation. Finally, the school can strengthen cooperation with the government and surrounding enterprises and establish a chain entrepreneurial education system of entrepreneurial training, project experiments, and entrepreneurial practice, so that students' entrepreneurial ability and entrepreneurial awareness can become a reality.

4.2. Analysis of Undergraduate EE. In this paper, through a survey of college students' cognition and interest in entrepreneurship, a questionnaire is used to send out questionnaires to the juniors and seniors of a certain university. When asked about "interest in entrepreneurship," 25\% of respondents chose "very interested," 32\% chose "relatively interested," 36\% chose "general," $5 \%$ chose "not very interested," and $2 \%$ chose "not interested." The results are shown in Table 2.

It can be seen that those surveyed with entrepreneurial intentions accounted for $60 \%$. In further investigation, it was found that men showed greater interest in entrepreneurship than women, junior college students showed greater interest in entrepreneurship than undergraduates, and undergraduates were more interested in entrepreneurship than graduate students. Among them, A means very interested, B means interested, $\mathrm{C}$ means generally interested, D means not interested, and E means very disinterested, as shown in Figure 3.

In the analysis of college students' entrepreneurial interest in Figure 3, it can be seen that, in the context of the Internet of Things, college students' learning interest has 


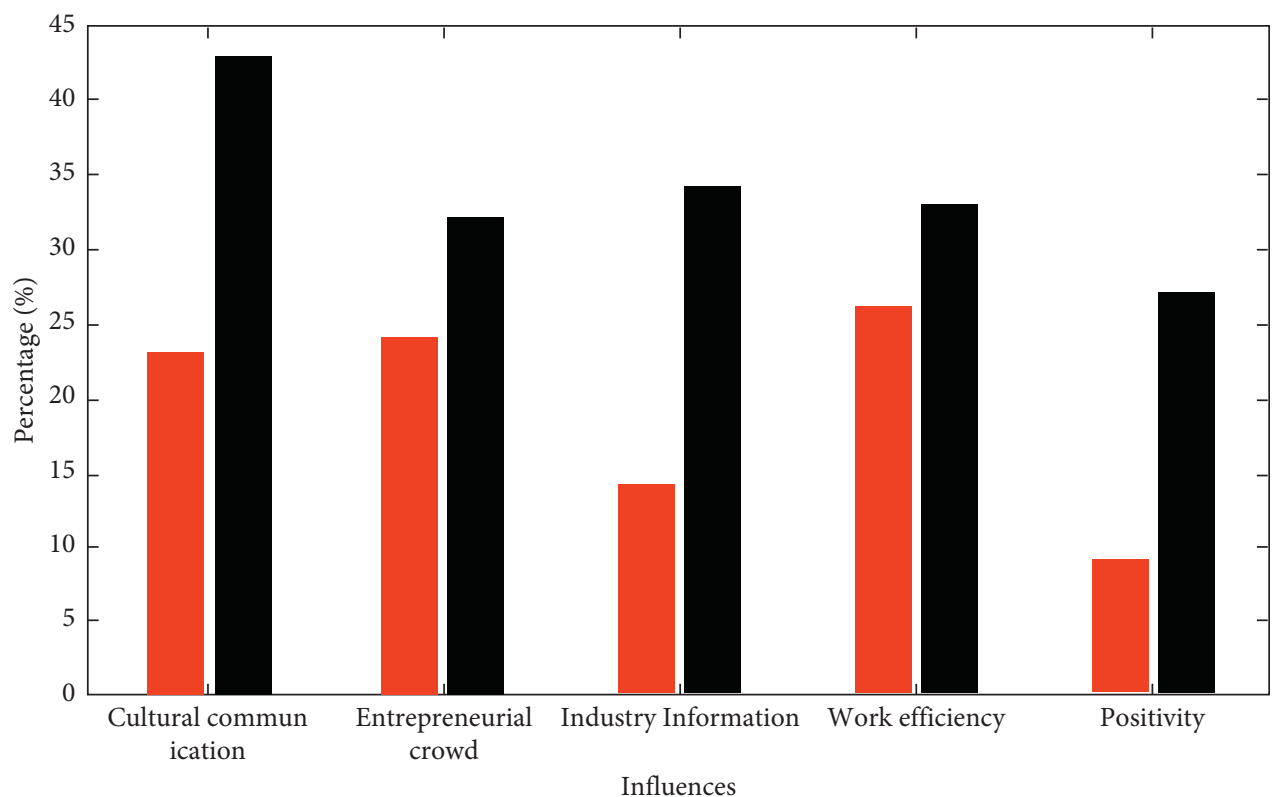

Before

After

Figure 2: Analysis of the impact of the IoT on college students' EE.

TABLE 2: Results of college students' interest in entrepreneurship.

\begin{tabular}{lccccc}
\hline Interest & Very interested & Interested & General & Not interested & Very not interested \\
\hline Number of people & 50 & 64 & 72 & 10 & 4 \\
Proportion & $25 \%$ & $32 \%$ & $36 \%$ & $5 \%$ & $2 \%$ \\
\hline
\end{tabular}

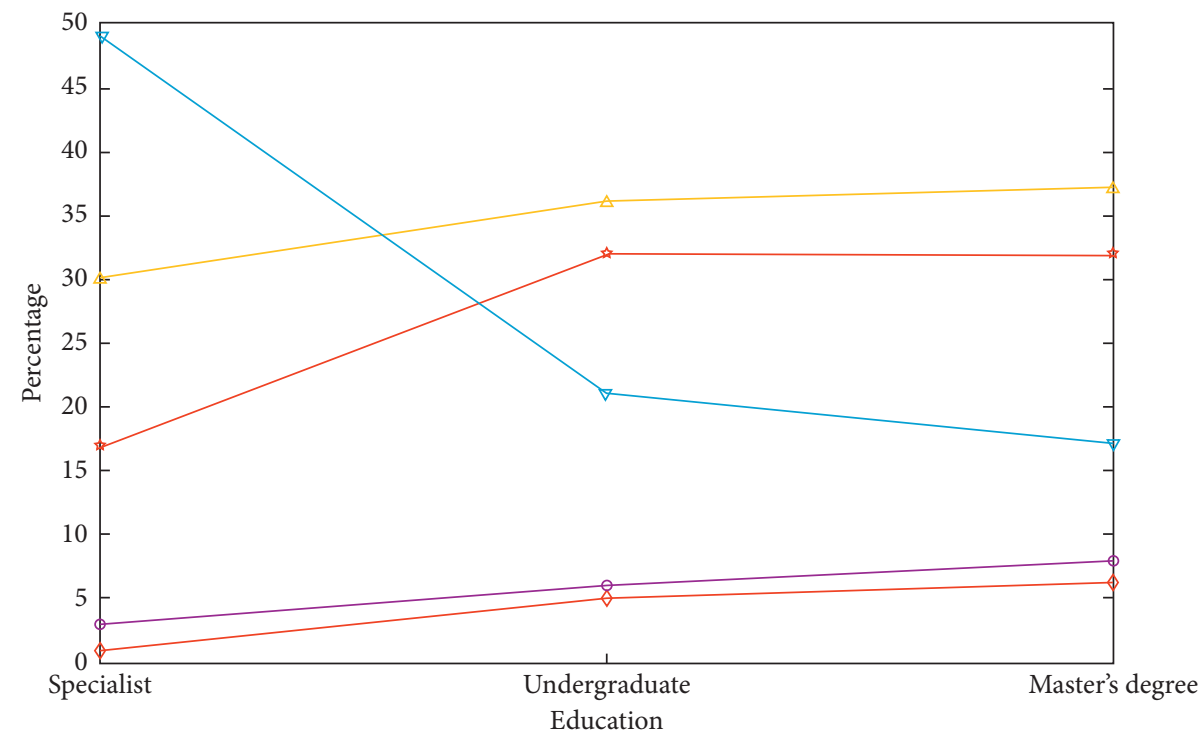

$$
\begin{array}{ll}
\rightarrow \mathrm{A} & \rightarrow \mathrm{D} \\
\rightarrow \mathrm{B} & \rightarrow \mathrm{E}
\end{array}
$$

FIGURE 3: Analysis of entrepreneurial interest of college students with different educational backgrounds. 


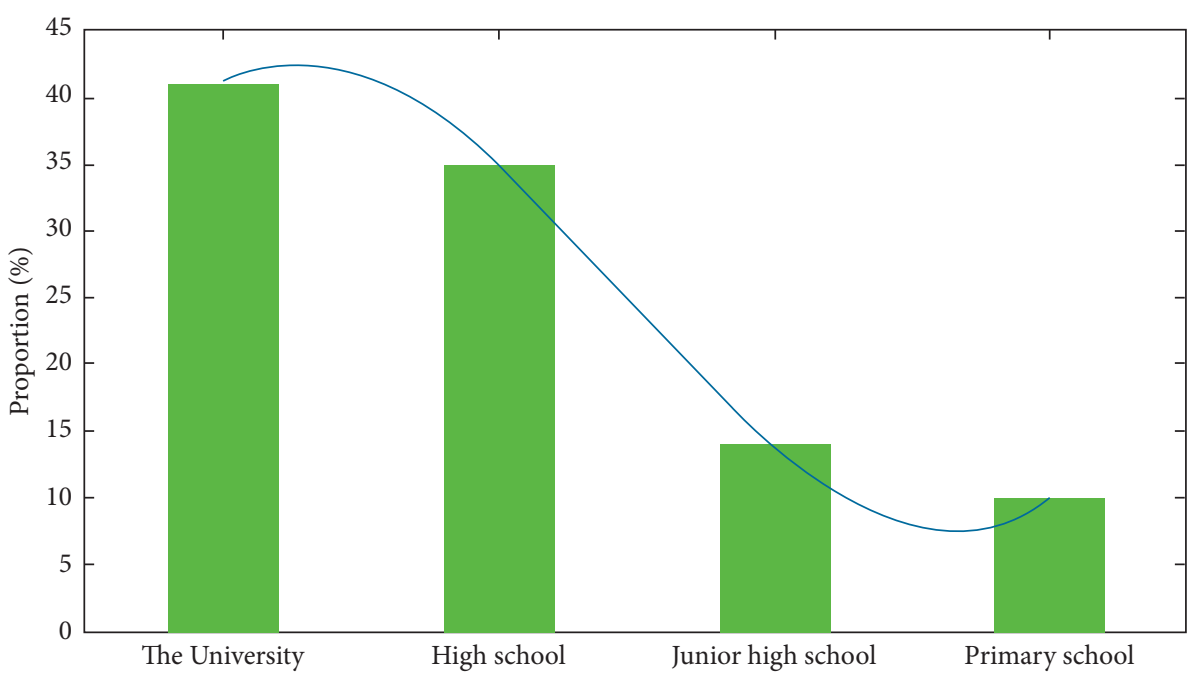

FIgURE 4: Time period analysis of undergraduate EE.

increased by about $15 \%$, which has fully aroused students' interest in learning and entrepreneurship.

In the "Reason for Entrepreneurship" survey, 54\% of the respondents chose "realize self-worth," $25 \%$ chose "to make big money," 14\% chose "accumulate experience," and 7\% chose "cannot find a suitable job." From the survey results, most students' entrepreneurial motives are correct, but there are still many students who regard entrepreneurship as a shortcut to making money and do not have a good understanding of the significance and value of innovation and $\mathrm{EE}$ in universities.

Regarding the "time to start receiving innovation and EE," $41 \%$ of the respondents believed that "university" should start, 30\% thought that it would start from "high school," $15 \%$ chose "junior high school," and only 14\% thought need to start from "primary school." The results show that most students' concepts of "lifelong education" and "education from childhood" have not yet been formed. The specific results are shown in Figure 4.

The investigation and analysis of the entrepreneurship education system for college students is mainly an investigation of educational concepts. The development of a correct educational learning concept can correct one's learning attitude and bring help to education and learning.

4.3. Analysis of College Students' EE System. The construction of the entrepreneurial education system is not only to provide students with entrepreneurial knowledge but also to improve the entrepreneurial structure. This is also an important channel to enhance students' entrepreneurial ability and a strong guarantee for colleges and universities to implement business training. Therefore, we must strive to establish and improve the corporate training curriculum system and incorporate it into the program. According to the characteristics of EE, this article divides $\mathrm{EE}$ into four modules, the proportions of which are shown in Figure 5.

The curriculum is based on the school's talent training goals and social development level and is based on scientific and cultural knowledge. In each professional learning plan,

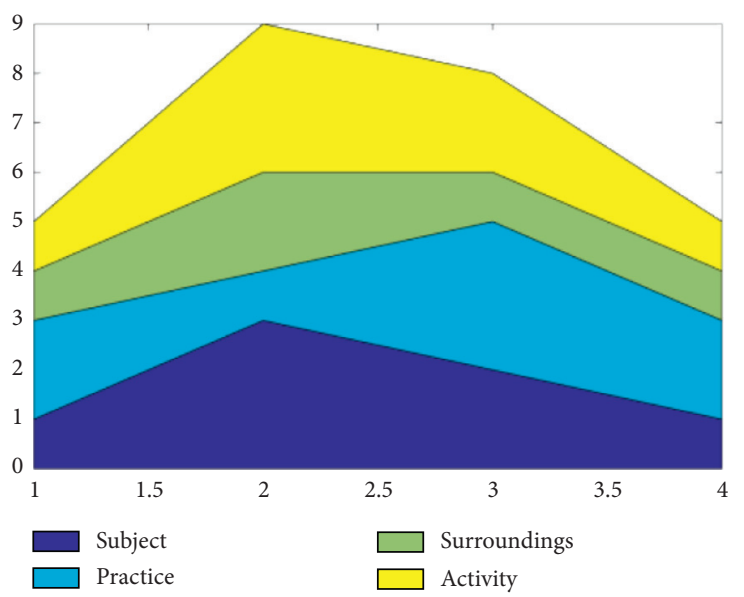

Figure 5: The proportion of undergraduate EE courses.

combining the objectives, content, and professional characteristics of the learning plan, infiltrating the content of EE, in order to let students understand entrepreneurship process, entrepreneurship training, theoretical knowledge, and entrepreneurial theoretical knowledge all offer theoretical courses, accounting for $3 / 10$ of the total. Entrepreneurship practice courses aim to improve students' business knowledge structure, participate in actual business management work, and train students in various aspects. A good entrepreneurial practice course can strengthen students' entrepreneurial abilities and skills and cultivate students' entrepreneurial personality. Entrepreneurship courses are the most important learning method in the structure of entrepreneurship learning courses, accounting for $1 / 4$ of the total.

Environmental curriculum refers to the school through various institutions and noninstitutions, explicit and implicit environmental factors, and their content, which influence the cultivation of the subtle consciousness and psychology of the educated and induce and shape the personality of entrepreneurial awareness and psychological quality levels. College 
environmental courses aim at $\mathrm{EE}$ and provide $\mathrm{EE}$ as the main purpose, creating a strong entrepreneurial cultural atmosphere in the university. EE courses do not have a unified teaching material, but through the enlightenment and pollution of entrepreneurial content, the training provided by this course can be felt in all fields, departments, and activities of the university, so that they can continuously improve and develop good business behavior habits, accounting for 1/5 of the total. EE courses at the university are carried out through various activities inside and outside the campus to broaden horizons, broaden knowledge, enrich experience, gain direct experience, understand business laws, understand the procedures and methods of business activities, and use a wide range of activities. This kind of knowledge solves business problems, focuses on the cultivation of entrepreneurial personality and entrepreneurial ability, and forms a complete knowledge structure and comprehensive ability training program. The purpose of activity courses is to fully and carefully develop the basic skills of students, accounting for $1 / 4$ of the total.

\section{Conclusions}

According to the current application status of the IoT, through the analysis of the architectural composition of the IoT based on the fusion of wireless transmission networks and wireless sensor networks and a detailed analysis of the characteristics and functions of its various components, the IoT sensor network, the IoT transmission network, and the IoT data center have been studied and elaborated separately, and the IoT has been analyzed and studied from the perspective of network functions.

The new education model also faces some problems, such as young people's lack of understanding of things, low acceptance, insufficient education atmosphere, and lack of relevant research. Colleges and universities must first raise teachers and students' awareness of the new model, create a good learning environment, organize a professional scientific research team, strive to promote innovation and cultivate students' entrepreneurial capabilities, and help students improve their entrepreneurial capabilities and practical skills.

The IoT is the dominant direction of the future communication industry, which will bring more convenience and changes to society. The architecture, general functions, key technologies, and network protocols of the IoT are the key foundation for the development of the IoT. The analysis and research on networking standardization and protocol design are not comprehensive enough. More detailed and reliable standard protocols need further analysis and research.

\section{Data Availability}

This paper does not cover data research. No data were used to support this study.

\section{Conflicts of Interest}

The authors declare that they have no conflicts of interest.

\section{Acknowledgments}

This research was supported by the following projects: the University-Level Research Team (Team no. XFU20KYTDC01) and the teaching team of the School of Innovation and Entrepreneurship (Team no. T1910) of Xi'an Institute of Translation, the Science and Technology Bureau of Chang'an District, 2017-2018 Science and Technology Research Project (Project no. cc1702), and Hangzhou Huizhuan Network Technology Co., Ltd., for cooperative education project of the Ministry of Education in 2018 (Project no. 19xyh021). This work was also supported by the research project on higher education teaching reform in Jilin Province in 2019, "Research on the Integration of Entrepreneurship Education and Professional Education in Local Colleges and Universities," with project leader Xiubo Liu.

\section{References}

[1] M. Zhou, Y. Wang, Z. Tian, Y. Lian, Y. Wang, and B. Wang, "Calibrated data simplification for energy-efficient location sensing in internet of things," IEEE Internet of Things Journal, vol. 6, no. 4, pp. 6125-6133, 2019.

[2] Z. Lv, R. Lou, and A. K. Singh, "AI empowered communication systems for intelligent transportation systems," IEEE Transactions on Intelligent Transportation Systems, vol. 22, 2020.

[3] D. Guido, H. Song, and A. Schmeink, Big Data Analytics for Cyber-Physical Systems: Machine Learning for the Internet of Things, Elsevier, Amsterdam, Netherlands, pp. 1-360, Article ID 9780128166376, 2019.

[4] S.-B. Tsai and K. Wang, "Using a novel method to evaluate the performance of human resources in green logistics enterprises," Ecological Chemistry and Engineering S, vol. 26, no. 4, pp. 629-640, 2019.

[5] Z. Lv, "Security of internet of things edge devices," Software: Practice and Experience, vol. 51, 2020.

[6] C. Perera, C. H. Liu, and S. Jayawardena, "The emerging IoT marketplace from an industrial perspective: a survey," IEEE Transactions on Emerging Topics in Computing, vol. 3, no. 4, pp. 585-598, 2017.

[7] Z. Lv and N. Kumar, "Software defined solutions for sensors in 6G/IoE," Computer Communications, vol. 153, pp. 42-47, 2020.

[8] T. Hasegawa and T. Maeda, "Development of full color LED display with color sensor function," Electronics and Communications in Japan, vol. 102, no. 3, pp. 21-28, 2019.

[9] H. Liao and J. Hitchcock, "Reported credibility techniques in higher education evaluation studies that use qualitative methods: a research synthesis," Evaluation and Program Planning, vol. 68, pp. 157-165, 2018.

[10] A. Alrawais, A. Alhothaily, C. Hu, and X. Cheng, "Fog computing for the IoT: security and privacy issues," IEEE Internet Computing, vol. 21, no. 2, pp. 34-42, 2017.

[11] H. Mostafa, T. Kerstin, and S. Regina, "Wearable devices in medical internet of things: scientific research and commercially available devices," Healthcare Informatics Research, vol. 23, no. 1, pp. 4-15, 2017.

[12] D. Mishra, A. Gunasekaran, and S. J. Childe, "Vision, applications and future challenges of Internet of Things: a bibliometric study of the recent literature," Industrial Management \& Data Systems, vol. 116, no. 7, pp. 1331-1355, 2017. 
[13] J. Lin, W. Yu, N. Zhang, X. Yang, H. Zhang, and W. Zhao, “A survey on internet of things: architecture, enabling technologies, security and privacy, and applications," IEEE Internet of Things Journal, vol. 4, no. 5, pp. 1125-1142, 2017.

[14] J. Singh, T. Pasquier, and J. Bacon, "Twenty security considerations for cloud-supported internet of things," IEEE Internet of Things Journal, vol. 3, no. 3, pp. 269-284, 2017.

[15] L. Wu, C.-H. Chen, and Q. Zhang, "A mobile positioning method based on deep learning techniques," Electronics, vol. 8, no. 1, Article ID 59, 2019.

[16] G. A. Akpakwu, B. J. Silva, G. P. Hancke, and A. M. AbuMahfouz, "A survey on $5 \mathrm{G}$ networks for the internet of things: communication technologies and challenges," IEEE Access, vol. 6, no. 12, pp. 3619-3647, 2018.

[17] M. Zhou, Y. Wang, Y. Liu, and Z. Tian, "An informationtheoretic view of WLAN localization error bound in GPSdenied environment," IEEE Transactions on Vehicular Technology, vol. 68, no. 4, pp. 4089-4093, 2019.

[18] Y. Yang, L. Wu, G. Yin, L. Li, and H. Zhao, "A survey on security and privacy issues in internet-of-things. Internet of Things Journal," IEEE Internet of Things Journal, vol. 4, no. 5, pp. 1250-1258, 2017.

[19] Z. Lv, H. A. N. Yang, A. K. Singh, G. Manogaran, and H. Lv, "Trustworthiness in industrial IoT systems based on artificial intelligence," IEEE Transactions on Industrial Informatics, vol. 17, 2020.

[20] A. Mosenia and N. K. Jha, "A comprehensive study of security of internet-of-things," IEEE Transactions on Emerging Topics in Computing, vol. 5, no. 4, pp. 586-602, 2017.

[21] D. Jiang, X. Ying, Y. Han, and Z. Lv, "Collaborative multi-hop routing in cognitive wireless networks," Wireless Personal Communications, vol. 86, no. 2, pp. 901-923, 2016.

[22] M. A. Razzaque, M. Milojevic-Jevric, and A. Palade, "Middleware for internet of things: a survey," IEEE Internet of Things Journal, vol. 3, no. 1, pp. 70-95, 2017.

[23] A. M. Rahmani, T. N. Gia, and B. Negash, "Exploiting smart e-health gateways at the edge of healthcare internet-of-things: a fog computing approach," Future Generation Computer Systems, vol. 78, no. 2, pp. 641-658, 2017.

[24] Y. Zhang and J. Wen, "The IoT electric business model: using blockchain technology for the internet of things," Peer-to-Peer Networking and Applications, vol. 10, no. 4, pp. 983-994, 2017.

[25] Z. Longchao, X. Jianjun, and Y. Limei, "Research on congestion elimination method of circuit overload and transmission congestion in the internet of things," Multimedia Tools and Applications, vol. 76, no. 17, pp. 18047-18066, 2017.

[26] W. C. Roberts, P. A. Grayburn, J. M. Guileyardo, and R. C. Stoler, "Full development of consequences of congenital pulmonic stenosis in eighty-four years," The American Journal of Cardiology, vol. 119, no. 8, pp. 1284-1287, 2017.

[27] A. Wilson, "Key learnings from first 2 years of a full-field css development in Oman," Journal of Petroleum Technology, vol. 69, no. 03, pp. 64-65, 2017.

[28] S.-B. Tsai, Y.-M. Wei, K.-Y. Chen, L. Xu, P. Du, and H.-C. Lee, "Evaluating green suppliers from green environmental perspective," Environment and Planning B: Planning and Design, vol. 43, no. 5, pp. 941-959, 2016.

[29] H. Chika, S. Natsumi, and S. Seung-Wook, "Ubiquitinproteasome system modulates zygotic genome activation in early mouse embryos and influences full-term development," Journal of Reproduction and Development, vol. 64, no. 1, pp. 65-74, 2017.

[30] M. Dório, D. J. Hunter, J. E. Collins et al., "Association of baseline and change in tibial and femoral cartilage thickness and development of widespread full-thickness cartilage loss in knee osteoarthritis-data from the osteoarthritis initiative," Osteoarthritis and Cartilage, vol. 28, no. 6, pp. 811-818, 2020.

[31] Z. Lv and A. K. Singh, "Big data analysis of internet of things system," ACM Transactions on Internet Technology, vol. 21, 2020.

[32] Z. Lv, R. Lou, J. Li, A. K. Singh, and H. Song, "Big data analytics for 6G-enabled massive internet of things," IEEE Internet of Things Journal, vol. 8, no. 99, p. 1, 2021.

[33] T. Hasegawa and T. Maeda, "Development of full color led display with color sensor function," IEEJ Transactions on Electronics, Information and Systems, vol. 138, no. 10, pp. 1176-1182, 2018.

[34] I. P. Bashilov, S. G. Volosov, V. A. Merkulov, N. P. Rybakov, S. Y. Sukonkin, and S. Y. Chervinchuk, "Development and full-scale testing of prototypes of tsdss-m and mdm digital seafloor seismic stations intended for security systems," Seismic Instruments, vol. 54, no. 6, pp. 619-625, 2018.

[35] M. Patzold, "Countdown for the full-scale development of $5 \mathrm{G}$ new radio," IEEE Vehicular Technology Magazine, vol. 13, no. 2, pp. 7-13, 2018.

[36] F. Rondina, S. Taddia, L. Mazzocchetti et al., "Development of full carbon wheels for sport cars with high-volume technology," Composite Structures, vol. 192, pp. 368-378, 2018.

[37] M. Hirohito, R. Asadur, and K. Hideki, "Current status of exposed endoscopic full-thickness resection and further development of non-exposed endoscopic full-thickness resection," Digestion, vol. 95, no. 1, pp. 6-15, 2017.

[38] W. Bowl, S. Schweinfurth, K. Holve, K. Stieger, B. Lorenz, and M. Andrassi-Darida, "Einfluss der Makulareifung auf Ganzfeld- und multifokales ERG bei ehemaligen Extremfrühgeborenen mit und ohne akute Frühgeborenenretinopathie," Klinische Monatsblätter für Augenheilkunde, vol. 234, no. 10, pp. 1235-1242, 2017. 